\title{
Pregnancy outcome in women with inflammatory bowel disease treated with anti-tumor necrosis factor and/or thiopurine therapy: a multicenter study from Japan
}

\author{
Shunsuke Komoto ${ }^{1}$, Satoshi Motoya ${ }^{2}$, Yuji Nishiwaki ${ }^{3}$, Toshiyuki Matsui ${ }^{4}$, Reiko Kunisaki ${ }^{5}$, Katsuyoshi \\ Matsuoka $^{6}$, Naoki Yoshimura ${ }^{7}$, Takashi Kagaya ${ }^{8}$, Makoto Naganuma ${ }^{9}$, Nobuyuki Hida ${ }^{10}$, Mamoru \\ Watanabe $^{6}$, Toshifumi Hibi ${ }^{11}$, Yasuo Suzuki ${ }^{12}$, Soichiro Miura ${ }^{13}$, Ryota Hokari ${ }^{1}$; Japanese study group for \\ pregnant women with IBD \\ ${ }^{1}$ Department of Internal Medicine, National Defense Medical College, Saitama, ${ }^{2}$ IBD Center, Sapporo Kosei General Hospital, Sapporo, \\ ${ }^{3}$ Department of Environmental and Occupational Health, School of Medicine, Toho University, Tokyo, ${ }^{4}$ Department of Gastroenterology and \\ Hepatology, Fukuoka University Chikushi Hospital, Fukuoka, ${ }^{5}$ IBD Center, Yokohama City University Medical Center, Yokohama, ${ }^{6}$ Department \\ of Gastroenterology and Hepatology, Tokyo Medical and Dental University, Tokyo, ${ }^{7}$ Department of Internal Medicine, Division of IBD, Tokyo \\ Yamate Medical Center, Tokyo, ${ }^{8}$ Department of gastroenterology, Kanazawa University Hospital, Ishikawa, ${ }^{9}$ Department of Gastroenterology \\ and Hepatology, Keio University School of Medicine, Tokyo, ${ }^{10}$ Division of Internal Medicine, Department of IBD, Hyogo College of Medicine, \\ Hyogo, ${ }^{11}$ Center for Advanced IBD Research and Treatment, Kitasato Institute Hospital, Tokyo, ${ }^{12}$ Department of Internal medicine, Sakura \\ Medical Center, Toho University, Sakura, ${ }^{13}$ Department of Internal Medicine, National Defense Medical College, Saitama, Japan
}

Background/Aims: Anti-tumor necrosis factor drugs (anti-TNF) and thiopurines are important treatment options in patients with inflammatory bowel disease (IBD), including during pregnancy. However, there are limited data on the benefit/risk profile of anti-TNF and thiopurines during pregnancy in Asia. The aim of this study was to analyze pregnancy outcomes of female Japanese IBD patients treated with anti-TNF and/or thiopurines. Methods: This cross-sectional study assessed pregnancy outcomes in 72 women with IBD. Pregnancy outcomes were compared among 31 pregnancies without exposure to infliximab (IFX), adalimumab (ADA), or thiopurines; 24 pregnancies with exposure to anti-TNF treatment (23 IFX, 1 ADA); 7 pregnancies with exposure to thiopurines alone; and 10 pregnancies with exposure to both IFX and thiopurines. Results: Thirty-five of the 41 pregnancies $(85.3 \%)$ that were exposed to anti-TNF treatment and/or thiopurines resulted in live births after a median gestational period of 38 weeks. Of the 35 live births, 3 involved premature deliveries; 7, low birth weight; and 1, a congenital abnormality. There were 6 spontaneous abortions in pregnancies that were exposed to anti-TNF treatment (17.7\%). Pregnancy outcomes among the 4 groups were similar, except for the rate of spontaneous abortions $(P=0.037)$. Conclusions: Exposure to anti-TNF treatment or thiopurines during pregnancy was not related to a higher incidence of adverse pregnancy outcomes in Japanese IBD patients except for spontaneous abortion. (Intest Res 2016;14:139-145)

Key Words: Inflammatory bowel diseases; Anti tumor necrosis factor-alpha; Thiopurines; Pregnancy outcome

Received September 15, 2015. Revised February 12, 2016

Accepted February 15, 2016.

Correspondence to Ryota Hokari, Department of Internal Medicine,

National Defense Medical College, 3-2, Namiki, Tokorozawa, Saitama 359-

8513, Japan. Tel: +81-4-2995-1609, Fax: +81-4-2996-5201,E-mail: ryota@

ndmc.ac.jp

Financial support: This study was supported in part by Health and Labour Sciences Research Grants for research on intractable diseases from the Ministry of Health, Labour and Welfare of Japan, and by grants from the National Defense Medical College. Conflict of interest: None.

\section{INTRODUCTION}

Onset of IBD, namely CD and UC, typically occurs during young adulthood, thus affecting individuals during their reproductive years. Procreation is one of the most important events in an individual's life. Naturally, IBD patients and their families are concerned about the effects of disease activity and drug exposures on fertility, pregnancy outcomes, and

\footnotetext{
๑ Copyright 2016. Korean Association for the Study of Intestinal Diseases. All rights reserved.

This is an Open Access article distributed under the terms of the Creative Commons Attribution Non-Commercial License (http://creativecommons.org/licenses/by-nc/4.0)

which permits unrestricted non-commercial use, distribution, and reproduction in any medium, provided the original work is properly cited.
} 
the developing child.

Recently, it was reported that anti-tumor necrosis factor drugs (anti-TNF) do not affect pregnancy outcomes in mothers with IBD, or their infants (congenital malformations and immunosuppression). ${ }^{1}$ Further, recent data have not identified any increased risk of infections during the first year of life in children born to mothers who have received biologics, even in combination with immunomodulators (thiopurines). ${ }^{2}$ By contrast, the use of thiopurines in pregnancy and their effects on pregnancy outcomes are still controversial. ${ }^{3}$ A systematic review showed that thiopurine exposure in women with IBD was not associated with low birth weight (LBW) or congenital abnormalities, but that it was associated with preterm birth. ${ }^{4}$

A recent study indicated that a non-Caucasian ethnicity is a predictor of adverse pregnancy outcomes in women with IBD. ${ }^{5}$ However, pregnancy outcomes of IBD patients treated with anti-TNF and/or thiopurines have not been investigated in Asian countries. Reconfirmation of the results of previous reports from western countries is important, because the genetic backgrounds and environmental factors influencing IBD differ between Asian and Western countries.

We hypothesized that pregnancy outcomes of IBD patients in Asian countries were associated with exposure to anti-TNF and/or thiopurines. The aim of this study was to analyze the pregnancy outcomes of female Japanese IBD patients.

\section{METHODS}

\section{Research Period and List of Collaborators}

Between September 2008 and March 2014, a multicenter, cross-sectional study was conducted.

Members of the Japanese study group for pregnant women with IBD are as follows (shown in alphabetical order of affiliation): Toshiyuki Matsui, Kozue Tsurumi (Gastroenterology and Hepatology, Fukuoka University Chikushi Hospital), Shinji Tanaka, Yoshitaka Ueno (Department of Endoscopy, Hiroshima University Hospital), Shiro Nakamura, Nobuyuki Hida (IBD Center, Hyogo College of Medicine), Takashi Kagaya, Kazuya Kitamura (Department of gastroenterology, Kanazawa university), Makoto Naganuma (Department of Gastroenterology and Hepatology, Keio University School of Medicine), Hiroshi Nakase, Minoru Matsuura, Toshifumi Hibi (Center for Advanced IBD Research and Treatment, Kitasato Institute Hospital), Takuya Yoshino (Department of Gastroenterology and Hepatology, Kyoto University Graduate School of Medicine), Motohiro Esaki (Department of
Medicine and Clinical Science, Graduate School of Medical Sciences, Kyushu University), Shunsuke Komoto, Soichiro Miura, Ryota Hokari Chie Kurihara, Hirokazu Sato, Masaaki Higashiyama, Kenichi Yoshikawa, Chikako Watanabe (Department of Internal Medicine, National Defense Medical College), Kenji Watanabe (Department of Gastroenterology, Osaka City University Graduate School of Medicine), Kenji Fujimori (Third Department of Internal Medicine, Saitama Medical School Moroyama), Satoshi Motoya, Hiroki Tanaka (IBD Center, Sapporo Kosei General Hospital), Shunji Ishihara (Department of Internal Medicine II, Shimane University Faculty of Medicine), Reiko Kunisaki (IBD Center, Yokohama City University Medical Center), Akira Sugita (Department of Surgery, Yokohama Municipal Citizen's Hospital), Yasuo Suzuki (Department of Internal medicine, Sakura Medical Center, Toho University ), Katsuyoshi Matsuoka, Mamoru Watanabe (Department of Gastroenterology and Hepatology, Tokyo Medical and Dental University), and Naoki Yoshimura (Department of Internal Medicine, Division of IBD, Tokyo Yamate Medical Center).

\section{Research Method}

A multicenter, cross-sectional study was conducted in Japan to investigate pregnancy outcomes of female Japanese IBD patients treated with anti-TNF and/or thiopurine therapy. The medical records and birth outcomes of 72 pregnancies in 72 women were analyzed retrospectively in 18 collaborating hospitals, in which the treating gastroenterologists were members of the Research Committee for Inflammatory Bowel Disease. From the medical records, the following information was collected: type of disease (UC or $\mathrm{CD}$ ), disease activity, and therapy. Each patient was given a questionnaire to answer. All data were anonymized and sent to an analysis center. Each patient completed a questionnaire, which gathered the following information regarding conception and pregnancy outcomes: smoking habits, alcohol consumption habits, and age at conception. Information was also requested regarding the outcome of the conceptions (live birth, spontaneous abortion, birth weight, and presence of congenital abnormalities). When women become pregnant in Japan, they receive a "notebook for mother and children" from regional health centers. Information regarding conception and the outcomes of the pregnancy was obtained from these notebooks. Information regarding pregnancy courses/outcomes and birth weight would be filled out by physicians or nurses in the notebooks. To identify the course and outcome of their pregnancies, patients 
referred to the notebooks. The study protocol was approved by the ethics committees at the National Defense Medical College (NDMC, No. 944) and was performed in accordance with the Declaration of Helsinki. Written informed consent was obtained from all subjects prior to participation.

\section{Categorization of Subgroups}

In the first analysis, pregnancies were classified into four groups depending on the drug exposure. Bio- IM-: pregnancies in women exposed to neither anti-TNF therapy or thiopurine therapy. Bio- IM+: pregnancies in women exposed to thiopurine therapy but not anti-TNF therapy. Bio+ IM-: pregnancies in women exposed to anti-TNF therapy but not thiopurine therapy. Bio+ IM+: pregnancies in women exposed to both anti-TNF therapy and thiopurine therapy.

In the second analysis, pregnancies were classified into 2 groups depending on the drug exposure. Bio-: pregnancies in women not exposed to anti-TNF therapy (alone or associated with thiopurines) Bio+: pregnancies in women exposed to anti-TNF therapy (alone or associated with thiopurines).

\section{Disease Activity and Medical Therapy}

Disease activity at the onset of pregnancy, medical therapy, and data regarding newborns were assessed retrospectively for each patient. The Harvey-Bradshaw index was calculated for CD activity and the partial Mayo score for UC activity.

\section{Definition of Terms for Pregnancy Outcomes}

LBW was defined as body weight less than $2500 \mathrm{~g}$ at birth.
Preterm birth was defined as birth at less than 35 weeks gestational age. Congenital anomalies were defined as structural or functional defects present at birth.

\section{Data Analysis and Statistical Methods}

The prevalences of adverse outcomes were analyzed in this study, including spontaneous abortion, preterm birth, LBW, and congenital malformations. Outcomes of pregnancies in women with IBD among the four groups of treatment (Bio- IM-, Bio only, IM only, and Bio+ IM+), were compared. For statistical analyses, Fisher's exact method for categorical variables and the Kruskal Wallis test for continuous variables were assessed using STATA version 12 (STATA Corporation, College Station, Texas, USA).

\section{RESULTS}

\section{Demographic Characteristics}

Thirty-one pregnancies received neither anti-TNF and/ or thiopurine therapy (Bio- IM-): 5 with CD, average age at delivery 34.4 [28-43] years; and 26 with UC, average age at delivery 31.5 [24-38] years. Twenty-four pregnancies received anti-TNF therapy only (Bio+): 24 with $\mathrm{CD}$, average age at delivery 30.0 [20-36] years; and none with UC. Seven pregnancies received thiopurine therapy only $(\mathrm{IM}+)$ : 4 with CD, average age at delivery 28.0 [24-35] years; and 3 with UC, average age at delivery 28.3 [19-35] years. Ten pregnancies received both anti-TNF and thiopurine therapy (Bio+ $\mathrm{IM}+$ ): 6 with CD, average age at delivery 30.5 [25-34] years; and 4 with UC, average age at delivery 35.3 [31-39] years. Smoking was not observed in the Bio- IM- group and was

Table 1. Characteristics of Pregnant Patients With IBD Included in the Study

\begin{tabular}{|c|c|c|c|c|c|}
\hline Variable & Bio- IM- ${ }^{a}(n=31)$ & Bio only $(n=24)$ & IM onlyc $(n=7)$ & Bio and $I^{d}{ }^{d}(n=10)$ & $P$-value ${ }^{\mathrm{e}}$ \\
\hline UC/UC+CD & 26/31 (83.9) & 0/24 (0) & $3 / 7$ (42.9) & 4/10 (40.0) & $<0.001$ \\
\hline Age & 30.0 & 30.9 & 30.7 & 31.9 & 0.269 \\
\hline Duration of disease (median) (yr) & 7.0 & 6.0 & 5.0 & 11.5 & 0.426 \\
\hline Smoking & 0/31 (0) & 4/24 (16.7) & $2 / 7$ (28.6) & $1 / 10(10.0)$ & 0.022 \\
\hline Drinking & 0/31 (0) & $2 / 24$ (8.3) & 0/7 (0) & $0 / 10(0)$ & 0.406 \\
\hline
\end{tabular}

Values are presented as $n(\%)$.

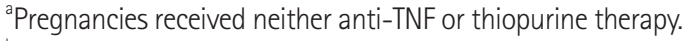

${ }^{b}$ Pregnancies received anti-TNF therapy only.

'Pregnancies received thiopurine therapy only.

${ }^{d}$ Pregnancies received both anti-TNF and thiopurine therapy.

eFisher's exact method for categorical variables and Kruskal Wallis test for continuous variables.

Bio, biologics; IM, immunomodulator. 
Table 2. Disease Activity in Women With IBD at the Onset of Pregnancy

\begin{tabular}{lccccc}
\hline \multicolumn{1}{c}{ Variable } & Bio- IM- $^{\text {a }}$ & Bio only $^{\text {b }}$ & IM only $^{\text {c }}$ & Bio and IM $^{\text {d }}$ & $\boldsymbol{P}_{\text {-value }}$ \\
\hline HBI score (No. of CD) & $2.6(5)$ & $1.9(24)$ & $3.6(4)$ & $4.2(6)$ & 0.067 \\
Partial Mayo score (No. of UC) & $0.9(26)$ & ND & $0.7(3)$ & $2.2(4)$ & 0.111 \\
\hline
\end{tabular}

${ }^{\text {a }}$ regnancies received neither anti-TNF or thiopurine therapy.

${ }^{b}$ Pregnancies received anti-TNF therapy only.

'Pregnancies received thiopurine therapy only.

${ }^{\mathrm{d}}$ Pregnancies received both anti-TNF and thiopurine therapy.

'Fisher's exact method for categorical variables.

Bio, biologics; IM, immunomodulator; HBI, Harvey-Bradshaw Index; ND, not done.

Table 3. Comparison of Pregnancy Outcomes in Patients With IBD Among Four Different Treatment Groups

\begin{tabular}{|c|c|c|c|c|c|}
\hline Variable & Bio- IM- ${ }^{a}(n=31)$ & Bio only ${ }^{b}(n=24)$ & IM onlyc $(n=7)$ & Bio and $I^{d}{ }^{d}(n=10)$ & P-value \\
\hline Premature birth & 2/29 (6.9) & $1 / 17$ (5.9) & 1/7 (14.3) & $1 / 8(12.5)$ & 0.618 \\
\hline Low birth weight & $7 / 29(24.1)$ & 4/17 (23.5) & $1 / 7(14.3)$ & $2 / 8(25.0)$ & 1.000 \\
\hline Birth weight (median) (g) & 2920 & 2844 & 2700 & 2738 & 0.886 \\
\hline Congenital anomaly & $2 / 31(6.5)$ & 0/24 (0) & 0/7 (0) & $1 / 10(10.0)$ & 0.457 \\
\hline Spontaneous abortion & 0/31 (0) & $4 / 24$ (16.7) & 0/7 (0) & $2 / 10(20.0)$ & 0.037 \\
\hline
\end{tabular}

Values are presented as $n(\%)$.

aPregnancies received neither anti-TNF or thiopurine therapy.

${ }^{b}$ Pregnancies received anti-TNF therapy only.

'Pregnancies received thiopurine therapy only.

${ }^{d}$ Pregnancies received both anti-TNF and thiopurine therapy.

${ }^{\text {e}}$ Fisher's exact method for categorical variables and Kruskal Wallis test for continuous variables.

Bio, biologics; IM, immunomodulator.

more commonly observed in the Bio+ group (16.7\%) and the $\mathrm{IM}+$ group (28.6\%). There was no difference in alcohol drinking habits among all groups. The above data regarding the patients' backgrounds are summarized in Table 1 .

\section{Therapy}

Among 41 pregnancies in women with IBD who were exposed to anti-TNF treatment and/or thiopurines, 22 cases received infliximab, 2 cases received adalimumab, 6 cases received azathioprine (25-100 mg), 1 case received 6-mercaptopurine $(60 \mathrm{mg})$, and 10 cases received a combination of infliximab and azathioprine. A combination of aminosalicylates plus anti-TNF therapy and/or thiopurines was used in 38 patients. A combination of oral prednisolone (5-30 mg) with infliximab and azathioprine was used in 2 cases. Of the 31 pregnancies in women with IBD who were not exposed to anti-TNF treatment or thiopurines, 28 received aminosalicylates. No patient received prednisolone treatment. Three patients received no therapy.

\section{Disease Activity at Onset of Pregnancy}

The mean Harvey-Bradshaw index at onset of pregnancy was 2.6 for the Bio- IM- group, 1.9 for Bio+ group, 3.6 for the $\mathrm{IM}+$ group, and 4.2 for the Bio+ IM+ group. There were no significant differences between all 4 groups $(P=0.067)$. Mean partial Mayo score at the onset of pregnancy was 0.9 in the Bio- IM- group, and 0.7 in IM+ and 2.2 in Bio+ IM+ groups. There were no significant differences between all 3 groups $(P=0.111)$. The above data regarding the disease activity at onset of pregnancy are summarized in Table 2.

\section{Pregnancy Outcomes}

Between the 4 groups, Bio-IM-, Bio+IM-, Bio-IM+, and Bio+IM+, a statistically significant difference was observed in spontaneous abortion rates (Table $3, P=0.037$ ). No significant differences were observed among the 4 groups concerning LBW, premature birth, birth weight, or congenital abnormality. Data of the pregnancy outcomes are summarized in Table 3. 
Table 4. Comparison of Pregnancy Outcomes in Patients With IBD Between the Group that Received Biologics Treatments and the Group Who Did not Receive Biologics Treatments

\begin{tabular}{lccc}
\hline \multicolumn{1}{c}{ Variable } & $\begin{array}{c}\text { TNF (-) } \\
(\mathbf{n = 3 8 )}\end{array}$ & $\begin{array}{c}\text { TNF (+) } \\
(\mathbf{n = 3 4 )}\end{array}$ & $\boldsymbol{P}^{\text {-value }}$ \\
\hline UC/UC+CD & $29 / 38(76.3)$ & $4 / 34(11.8)$ & $<0.001$ \\
Premature birth & $3 / 36(8.3)$ & $2 / 25(8.0)$ & 1.000 \\
Low birth weight & $8 / 36(22.2)$ & $6 / 25(24.0)$ & 1.000 \\
Birth weight (median) (g) & 2890 & 2844 & 0.655 \\
Congenital anomaly & $2 / 38(5.3)$ & $1 / 34(2.9)$ & 1.000 \\
Spontaneous abortion & $0 / 38(0)$ & $6 / 34(17.7)$ & 0.009 \\
\hline
\end{tabular}

Values are presented as $n(\%)$.

${ }^{a}$ Fisher's exact method for categorical variables and Kruskal Wallis test for continuous variables.

TNF, tumor necrosis factor.

Comparing the 2 groups Bio- and Bio+, a statistically significant difference was observed in spontaneous abortion rates between the 2 groups (Table $4, P=0.009$ ). No significant differences were observed between the 2 groups concerning LBW, premature birth, birth weight, or congenital abnormality. Data of the pregnancy outcomes are summarized in Table 4.

\section{DISCUSSION}

Data on drug safety during pregnancy are largely restricted to the cumulative experience of patients and physicians and are often limited to case reports. Previous investigations of pregnancy outcomes in women with IBD are mainly limited to European populations. To our knowledge, this is the first Asian multicenter cross sectional study examining the outcomes of pregnancies in IBD patients treated with anti-TNF and/or thiopurine therapy.

Because pregnancy outcome is highly influenced by age, ${ }^{6}$ the ages of the patients were compared. There was no significant difference among 4 groups. In addition, disease activity has been associated with negative fetal outcomes in a number of reports. ${ }^{7-11}$ Meta-analysis has shown that disease activity at conception affects disease course during pregnancy. ${ }^{12}$ Thus, to determine the severity of disease in each patient, the Harvey-Bradshaw index was calculated for those with $\mathrm{CD}$ and the partial Mayo score was calculated for those with UC at the time of conception. There were no significant differences between all 4 groups of patients. LBW and the incidences of congenital malformations and childhood diseases did not increase in women who were exposed to anti-TNF or thiopurines during their pregnancies compared to those who were not exposed. The average gestational age of babies in the 4 groups, Bio-IM-, Bio+IM-, Bio-IM+, and Bio+IM+ were 30.0 weeks, 30.9 weeks, 30.7 weeks, and 31.9 weeks, respectively.

In Akbari's study, thiopurine exposure in women with IBD was associated with preterm birth. ${ }^{4}$ In our study, there was no increase in the incidence of preterm birth in pregnancies exposed to thiopurines. However, it is possible that we could not detect small differences because of the small sample size of this study. The incidences of LBW, preterm birth, and congenital malformation are $6.4 \%, 2.1 \%$, and $1 \%-4 \%$ respectively in the normal population. ${ }^{13}$ In our analysis, the incidences of LBW, preterm birth, and congenital malformation in those who received either anti-TNF-alpha antibody or thiopurines were $17 \%(7 / 41), 7.3 \%(3 / 41)$, and $2.4 \%(1 / 41)$. On the other hand, the incidences of LBW, preterm birth, and congenital malformations in the group who had not received antiTNF-alpha antibodies or thiopurines were $24 \%$ (7/29), $6.9 \%$ (2/29), and 6.5\% (2/31). Incidences of LBW and preterm birth were higher in our study groups than in the general population regardless of the use of anti-TNF-alpha antibody or thiopurines, suggesting that IBD itself seems to be an independent risk factor for an abnormal outcome concerning LBW and preterm birth. Incidence of congenital malformation was comparable between the general population, those who received either anti-TNF-alpha antibodies or thiopurines, and those who did not receive anti-TNF-alpha antibody or thiopurines. Thus, we could not detect any proof of exposure to anti- TNF-alpha antibody or thiopurines being a risk factor for LBW, preterm birth, and congenital malformations. Our data support that medical maintenance treatment should be continued during pregnancy to avoid relapses.

In our analysis, spontaneous abortion occurred more commonly in the group exposed to anti-TNF-alpha antibodies. We observed significant differences when we divided the groups into 4 as well as into 2, as a comparison between patients who were treated with anti-TNF and who were not treated with anti-TNF. Another Japanese study reported that the incidences of spontaneous abortion (OR, 5.3; 95\% CI, 1.1-25.0) were significantly higher in CD patients whose conceptions occurred after disease onset than in those who had conceived prior to disease onset. In that report, 3 patients had received anti-TNF-alpha antibody (infliximab). ${ }^{14}$ In our study, most patients treated with anti-TNF had CD (88\%). Because the number of patients with UC who were treated with anti-TNF was small, we cannot conclude whether antiTNF treatment was a risk factor for spontaneous abortion in the Japanese population. Although statistically nonsignifi- 
cant, disease activity scores were higher in the anti-TNF- and thiopurine therapy-exposed groups. Whether this association is secondary to the use of anti-TNF or whether use of anti-TNF is only a marker for more severe disease is unclear. Johnson et al. reported outcomes of pregnancies in women with IBD treated with anti-TNF therapy from a Western country and showed that it may increase the risk of spontaneous abortion (exposed 5/34 vs. never exposed 3/55; OR, 2.99). ${ }^{15}$ In contrast, Schnitzler reported that the abortion rate in a TNF -exposed group vs. a non-exposed group was not significantly different (exposed 9/42 vs. non-exposed 12/78; OR, 1.5 [0.57 to 3.92$]$ ). ${ }^{2}$ In a systematic review and meta-analysis on the effects of anti-TNF-alpha antibodies on female patients with IBD, there was no increase in the occurrence of adverse pregnancy outcomes including spontaneous abortion in comparison with that reported in controls, except for the significant decrease in gestational age of newborns of drug-exposed mothers in 1 trial. ${ }^{16}$ Thus, the use of anti-TNF during pregnancy in IBD patients from Western countries appears to be acceptable. However, the pregnancy outcomes of patients with rheumatoid arthritis (another auto-immune disease treated with anti- TNF therapy) showed that the rate of spontaneous abortion was highest among patients exposed to anti-TNF at the time of conception (with methotrexate/leflunomide $33 \%$ and without methotrexate/leflunomide $24 \%$ ). This compared with $10 \%$ spontaneous abortions in the control group. In that report, there was no significant increase in congenital malformations or premature birth. ${ }^{17}$ There was 1 case report of spontaneous abortion during use of ustekinumab, an anti-TNF agent, in women with psoriatic arthritis. $^{18}$ Thus, concerning spontaneous abortion rates in IBD patients of Asian descent, no firm conclusions can be drawn about the safety of anti-TNF during pregnancy.

There are several limitations in our study. The number of patients was limited. Each drug exposure group included a mixture of CD and UC patients, and ratios were different in each group. Disease activity of each group was not matched.

In conclusion, exposure to anti-TNF treatment or thiopurines during pregnancy in IBD patients of Asian descent was not related to a higher incidence of adverse pregnancy outcomes in IBD patients except for spontaneous abortion. There is a possibility that the increased spontaneous abortion rate was associated with anti-TNF use. However, because the number of patients in our study is limited, a further large-scale study is necessary.

\section{REFERENCES}

1. Nielsen OH, Loftus EV Jr., Jess T. Safety of TNF- $\alpha$ inhibitors during IBD pregnancy: a systematic review. BMC Med 2013;11:174. doi: 10.1186/1741-7015-11-174.

2. Schnitzler F, Fidder H, Ferrante M, et al. Outcome of pregnancy in women with inflammatory bowel disease treated with antitumor necrosis factor therapy. Inflamm Bowel Dis 2011;17:1846-1854.

3. Coelho J, Beaugerie L, Colombel JF, et al. Pregnancy outcome in patients with inflammatory bowel disease treated with thiopurines: cohort from the CESAME Study. Gut 2011;60:198-203.

4. Akbari M, Shah S, Velayos FS, Mahadevan U, Cheifetz AS. Systematic review and meta-analysis on the effects of thiopurines on birth outcomes from female and male patients with inflammatory bowel disease. Inflamm Bowel Dis 2013;19:15-22.

5. Mahadevan U, Sandborn WJ, Li DK, Hakimian S, Kane S, Corley DA. Pregnancy outcomes in women with inflammatory bowel disease: a large community-based study from Northern California. Gastroenterology 2007;133:1106-1112.

6. Ziadeh S, Yahaya A. Pregnancy outcome at age 40 and older. Arch Gynecol Obstet 2001;265:30-33.

7. Bush MC, Patel S, Lapinski RH, Stone JL. Perinatal outcomes in inflammatory bowel disease. J Matern Fetal Neonatal Med 2004;15:237-241.

8. Fedorkow DM, Persaud D, Nimrod CA. Inflammatory bowel disease: a controlled study of late pregnancy outcome. Am J Obstet Gynecol 1989;160:998-1001.

9. Morales M, Berney T, Jenny A, Morel P, Extermann P. Crohn's disease as a risk factor for the outcome of pregnancy. Hepatogastroenterology 2000;47:1595-1598.

10. Nørgård B, Hundborg HH, Jacobsen BA, Nielsen GL, Fonager K. Disease activity in pregnant women with Crohn's disease and birth outcomes: a regional Danish cohort study. Am J Gastroenterol 2007;102:1947-1954.

11. Reddy D, Murphy SJ, Kane SV, Present DH, Kornbluth AA. Relapses of inflammatory bowel disease during pregnancy: inhospital management and birth outcomes. Am J Gastroenterol 2008;103:1203-1209.

12. Abhyankar A, Ham M, Moss AC. Meta-analysis: the impact of disease activity at conception on disease activity during pregnancy in patients with inflammatory bowel disease. Aliment Pharmacol Ther 2013;38:460-466.

13. Cornish J, Tan E, Teare J, et al. A meta-analysis on the influence of inflammatory bowel disease on pregnancy. Gut 2007;56:830-837.

14. Naganuma M, Kunisaki R, Yoshimura N, et al. Conception and pregnancy outcome in women with inflammatory bowel disease: a multicentre study from Japan. J Crohns Colitis 2011;5:317-323 
15. Johnson D, Jones K, Jimenez J, Mirrasoul N, Salas E, Chambers C. Pregnancy outcomes in women exposed to adalimumab: an update on the autoimmune diseases in Pregnancy Project. Am J Gastroenterol 2009;104:S410.

16. Mozaffari S, Abdolghaffari AH, Nikfar S, Abdollahi M. Pregnancy outcomes in women with inflammatory bowel disease following exposure to thiopurines and antitumor necrosis factor drugs: a systematic review with meta-analysis. Hum Exp Toxicol 2015;34:445-459.
17. Verstappen SM, King Y, Watson KD, Symmons DP, Hyrich KL; BSRBR Control Centre Consortium, BSR Biologics Register. Anti-TNF therapies and pregnancy: outcome of 130 pregnancies in the British Society for Rheumatology Biologics Register. Ann Rheum Dis 2011;70:823-826.

18. Fotiadou C, Lazaridou E, Sotiriou E, Ioannides D. Spontaneous abortion during ustekinumab therapy. J Dermatol Case Rep 2012;6:105-107. 